\title{
Tratamiento de la Inecuación en el Currículum y Textos Escolares Chilenos
}

\section{Inequalities Handling in Chilean Curriculum and School Texts}

\author{
Yerka Monje* \\ ORCID iD 0000-0003-4075-1688 \\ María José Seckel $^{* *}$ \\ ORCID iD 0000-0001-7960-746X \\ Adriana Breda ${ }^{* * *}$ \\ ORCID iD 0000-0002-7764-0511
}

\begin{abstract}
Resumen
En este trabajo se realiza un análisis del tratamiento de la inecuación, tanto en el currículum, a través de sus planes y programas, como en los textos escolares chilenos. Se trata de una investigación exploratoria que sigue una metodología de análisis cualitativa, donde el objeto matemático inecuación será planteado con un esquema de complejidad de elaboración propia, que permite un análisis visual de lo que ocurre en Chile. Dentro de los hallazgos, revelamos algunos quiebres en la progresión de la enseñanza del objeto matemático en estudio, es decir, el tratamiento que se le da al objeto en los diferentes cursos no lo abarcaría en su totalidad, estas podrían ser atendidas en la planificación de aula de cada profesor con el objetivo de mejorar los procesos de aprendizaje. Cabe destacar que el proceso de estudio que se muestra en este trabajo puede ser replicado en el análisis de otros objetos matemáticos.
\end{abstract}

Palabras Clave: Inecuación. Textos Escolares. Currículum de Matemáticas. Complejidad Matemática.

\begin{abstract}
In this paper, we make an analysis of inequality handling, including both: the Chilean curriculum, through its plans and programs, and school texts. It has been decided to work on an exploratory research that follows a methodology of qualitative analysis, where the mathematical object will be equated with a complexity scheme of its own, allowing a visual analysis of what happens in Chile. Within the findings, we reveal some differences in the teaching progression for mathematical object under study, that is to say, the treatment given to the object in different courses would not cover it in its entirety. The missing contents could be addressed in classroom planning for every teacher
\end{abstract}

\footnotetext{
* Licenciada en Educación por la Universidad de Concepción (UDEC). Estudiante de Magíster en Didáctica de la Matemática en la Universidad Católica de la Santísima Concepción (UCSC), Concepción, Chile. Dirección postal: Alonso de Rivera, 2050, Concepción, Chile, CEP: 4030-000. E-mail: ymonje @ magister.ucsc.cl.

** Doctora en Formación del Profesorado: práctica educativa y comunicación, Universidad de Barcelona (UB). Profesora de la Universidad Católica del Maule (UCM), Talca, Región del Maule y Chile. Dirección postal: Departamento de formación Inicial Escolar, Facultad de Ciencias de la Educación, Avenida San Miguel 3605, Talca, Chile, CEP: 3460-000. E-mail: mjseckel@ucm.cl.

*** Doctora en Educación en Ciencias y Matemáticas por la Pontifícia Universidade Católica do Rio Grande do Sul (PUCRS). Investigadora postdoctoral de la Universidad de Barcelona (UB), Barcelona, España. Dirección postal: Departamento de Educación Lingüística y Literaria y de Didáctica de las CCEE y de la Matemática, Facultad de Educación, Passeig de la Vall d'Hebrón, 171, Barcelona, España, CEP: 08035. E-mail: adriana.breda@gmail.com.
} 
with the aim of improving learning processes. Moreover, it is important to mention that the study process explained in this research can be also applied in the analysis of other mathematical objects.

Keywords: Inequality. School Texts. Mathematics Curriculum. Mathematical complexity.

\section{Introducción}

El rol de las desigualdades y de las inecuaciones es fundamental en la ciencia y tecnología. Estos objetos matemáticos están íntimamente ligados al concepto de aproximación. El gran matemático y filósofo inglés Russell (STEFFENS, 2006, p. 20) indicó lo siguiente: "Toda ciencia exacta es dominada por la idea de aproximación". Cada vez que uno computa, uno aproxima y, cada vez que uno aproxima, uno compara y es ahí donde entran en juego las desigualdades o inecuaciones.

En la vida cotidiana también es de suma importancia saber comparar números para tomar decisiones, por ejemplo, para ver qué tasa de interés nos conviene al pedir un préstamo, o en el solo hecho de comprar un auto uno compara el rendimiento en carretera o en ciudad; cantidad de puertas; espacio en el maletero etc. Por ello, se puede concluir que la comparación de números, las desigualdades y las inecuaciones deben formar parte del conocimiento matemático que todo ciudadano debe saber.

En esta línea, Paulos (1990) presenta problemas para entender la matemática en el contexto de la vida cotidiana y, a partir de ejemplos y casos reales, explica cómo el común de los ciudadanos tiende a malinterpretar lo que ve y oye. Además, insiste en la importancia del conocimiento matemático (mínimo) que cada persona debe tener y hace varias propuestas para mejorar tal entendimiento. Él introdujo el término anumerismo (innumerancy, en inglés) que significa la incapacidad de comprender conceptos matemáticos aplicados en la vida cotidiana, dando algunos ejemplos de esta condición a partir de situaciones relacionadas con las desigualdades o inecuaciones cotidianas. En esta línea, Cerda et al. (2011) plantean que, para mejorar el nivel de competencia matemática, se requiere de un proceso de enseñanza y aprendizaje progresivo, donde se considere la complejidad del objeto matemático a enseñar.

Estos antecedentes despiertan un interés por conocer el tratamiento de la inecuación en el contexto escolar chileno, específicamente, nos ha llevado a analizar el currículum nacional y los textos de estudio que son distribuidos gratuitamente por el Ministerio de Educación de Chile (MINEDUC) para conocer cómo se ha planificado la enseñanza de dicho objeto matemático y, a partir de ello, determinar si ésta se ha planteado de manera progresiva o no. En definitiva, el trabajo que se presenta corresponde a un primer acercamiento a la temática, donde se pretende 
llevar a cabo un análisis general de la cuestión para, posteriormente, centrar el análisis en los tipos de tareas propuestos.

\section{Marco de referencia}

En el ámbito de la didáctica de la matemática, diversos estudios se han interesado en analizar el tratamiento que se le da a determinados objetos matemáticos, tanto en el currículum como en los textos escolares (Por ejemplo: RICO, 1998; PINO; BLANCO, 2008; PINO-FAN et al., 2013; PARRA; PINO-FAN, 2017). Los trabajos desarrollados en esta línea, permiten distinguir la necesidad de contar con un referente que, a partir de la comparación, nos permita visualizar o evidenciar si se contemplan, o no, todos los componentes de un determinado objeto matemático.

En Breda, Font y Pino-Fan (en prensa) se plantea la discusión de que, con el objetivo de mejorar los procesos de enseñanza y aprendizaje de las matemáticas, en el marco del Enfoque Ontosemiótico de la Cognición e Instrucción Matemática (EOS) se han desarrollado distintas herramientas de análisis de la actividad matemática.

Una de esas herramientas son los criterios de idoneidad didáctica. Se entiende por idoneidad didáctica de un proceso de enseñanza-aprendizaje al grado en que éste (o una parte del mismo) reúne ciertas características que permiten calificarlo como óptimo o adecuado para conseguir la adaptación entre los significados personales logrados por los estudiantes (aprendizaje) y los significados institucionales pretendidos o implementados (enseñanza), teniendo en cuenta las circunstancias y recursos disponibles (entorno).

Es en Godino (2003) y en Godino, Wilhelmi y Bencomo (2005) donde se hace mención, por primera vez, a la noción de idoneidad didáctica. En Godino et al. (2006) es donde se comienza a desarrollar y precisar la noción de idoneidad didáctica, descomponiéndola en seis idoneidades específicas:

1. Idoneidad epistémica, para valorar si las matemáticas que se enseñan son unas "buenas matemáticas". 2. Idoneidad cognitiva, para valorar, antes de iniciar el proceso de instrucción, si lo que se quiere enseñar está a una distancia razonable de lo que saben los alumnos y, después del proceso, si los aprendizajes logrados se acercan a los que se pretendían enseñar. 3. Idoneidad interaccional, para valorar si la interacción ha resuelto dudas y dificultades de los alumnos. 4. Idoneidad mediacional, para valorar la adecuación de recursos materiales y temporales utilizados en el proceso de instrucción. 5. Idoneidad emocional, para valorar la implicación (interés, motivación) de los alumnos en el proceso de instrucción. 6. Idoneidad ecológica, para valorar la adecuación del proceso de instrucción al proyecto educativo del centro, las directrices curriculares, las condiciones del entorno social y profesional etc. (FONT; PLANAS; GODINO, 2010, p.101). 
La operatividad de los criterios de idoneidad exige definir un conjunto de indicadores observables, que permitan valorar el grado de idoneidad de cada una de las facetas del proceso de estudio. Por ejemplo, todos concordamos que es necesario implementar unas buenas matemáticas, pero podemos entender cosas muy diferentes por buenas matemáticas. Godino et al. (2007) explican que, para algunos criterios, los indicadores son relativamente fáciles de consensuar (por ejemplo, para el criterio de idoneidad de medios), para otros, como es el caso de la idoneidad epistémica es más difícil.

Por ejemplo, se puede aumentar la idoneidad epistémica presentando a los alumnos una muestra representativa, variada y articulada de situaciones-problema (contextualizados, con diferentes niveles de dificultad etc.); buscando explorar el uso de los modos de comunicación (expresión verbal, gráfica, simbólica etc.), y las conversiones que pueden surgir entre ellos; adecuando el lenguaje matemático y la claridad y corrección de definiciones y procedimientos conforme al nivel educativo en que se está trabajando; presentando los resultados enunciados básicos del tema y adecuando explicaciones, comprobaciones y demostraciones dentro del nivel escolar al que se dirige y estableciendo relaciones entre definiciones, propiedades, problemas del tema estudiado. En este sentido, para que se logre la idoneidad epistémica es pertinente contemplar, en el proceso de instrucción, los componentes e indicadores presentes en el Cuadro 1 .

\begin{tabular}{|ll|}
\hline Componente & Indicador \\
\hline Errores & No se observan prácticas que se consideren incorrectas desde el punto de vista \\
& matemático. \\
\hline \multirow{3}{*}{ Ambigüedades } & No se observan ambigüedades que puedan llevar a la confusión a los alumnos: \\
& definiciones y procedimientos clara y correctamente enunciados, adaptados al \\
& nivel educativo al que se dirigen; adecuación de las explicaciones, \\
& comprobaciones, demostraciones al nivel educativo a que se dirigen, uso \\
& controlado de metáforas, etc. \\
\hline \multirow{2}{*}{ Riqueza de procesos } & La secuencia de tareas contempla la realización de procesos relevantes en la \\
& actividad matemática (modelización, argumentación, resolución de problemas, \\
& conexiones, etc.). \\
\hline Los significados parciales implementados (definiciones, propiedades, \\
procedimientos, etc.) son una muestra representativa de la complejidad de la \\
noción matemática que se quiere enseñar contemplada en el currículo. \\
Los significados parciales implementados (definiciones, propiedades, \\
procedimientos, etc.) son una muestra representativa de la complejidad de la \\
noción matemática que se quiere enseñar. \\
Para los significados parciales implementados, muestra representativa de \\
problemas. \\
Para los significados parciales implementados, uso de diferentes modos de \\
expresión (verbal, gráfico, simbólico...), tratamientos y conversiones entre los \\
mismos.
\end{tabular}


En el marco del EOS, se plantea la idea de idoneidad epistémica como una herramienta útil para analizar el grado de representatividad de los significados institucionales implementados (o pretendidos), respecto de un significado de referencia (GODINO, 2013). En esta línea, el estudio que se presenta se ha focalizado en algunos de los indicadores del componente representatividad de la idoneidad epistémica del objeto matemático inecuación para lo cual se requiere la caracterización de los significados parciales que corresponden a una muestra representativa de lo que se quiere enseñar en el contexto de complejidad matemática de dicho objeto (FONT; BREDA; SECKEL, 2017).

Para lo anterior, es posible considerar la idea de complejidad matemática desarrollada por Rondero y Font (2015) en el estudio de la media aritmética y aplicada en Seckel (2016) para el estudio de la proporcionalidad. El concepto de complejidad de un determinado objeto matemático es entendido como la suma de todos los componentes de un determinado objeto y las conexiones que existen entre ellos. En base a esta idea, la complejidad del objeto matemático en estudio será vista desde la dualidad unitaria-sistémica, por ejemplo (en el contexto escolar chileno), en el estudio de la función raíz cuadrada, desde séptimo básico (12 a 13 años) hasta segundo medio (15 a 16 años) se ven las raíces cuadradas, su cálculo y propiedades, en tercero medio (16 a 17 años) todos estos elementos se consideran algo conocido y, en consecuencia, como entidades unitarias (elementales).

Este mismo objeto matemático, en los cursos de séptimo básico y segundo medio, es considerado de manera sistémica para su aprendizaje. Una analogía parecida es la que presentan Rondero y Font (2015, p. 30), donde concluyen que "un elemento esencial para que sea posible esta mirada dual (unitaria-sistémica) hacia los objetos matemáticos es que los componentes que resultan de la mirada compleja se articulan (conectan) entre sí, posibilitando la mirada unitaria del objeto matemático".

Con la finalidad de construir un referente que nos permitiese visualizar la complejidad matemática de la inecuación (o desigualdad), se ha realizado una búsqueda bibliográfica que nos da cuenta del escaso desarrollo histórico-epistémico de este objeto de estudio. Esta misma dificultad fue manifestada por Borello y Lezama (2009, p. 1093) quienes declaran que, de las investigaciones consultadas:

Todas tienen un corte de tipo cognitivo y/o didáctico, es decir, buscan entender qué es lo que obstaculiza el correcto aprendizaje de las inecuaciones y qué es lo que lo podría facilitar para intentar llevarlo al aula a fin de mejorar los procesos de enseñanza y el aprendizaje de los alumnos.

Dichos hallazgos, que van en la misma línea de los encontrados en este estudio (VARGAS，2013; GARROTE; HIDALGO; BLANCO，2004; VRANCKEN; ENGLER; 
MÜLLER, 2010), se enfocan en la dificultad del aprendizaje de la inecuación y en cómo facilitar su estudio. Lo más cercano a antecedentes históricos se relaciona más bien con antecedentes generales del álgebra. Como ejemplo de ello, tenemos lo planteado por Baldor (1988) y Stewart (2008), quienes indican que los primeros documentos de álgebra tienen una antigüedad de unos 4000 años, los cuales se encontraron en Egipto y Babilonia, pues fueron estas dos civilizaciones las que le otorgaron una importancia selecta al tema, lo que permite concluir que se necesitaron cientos de año para llegar a alcanzar el simbolismo algebraico actual.

Otros antecedentes que menciona Stewart (2008, p. 66) es que "la palabra "álgebra" procede del árabeal-jabr, un término empleado por Muhammad ibn Musa al-Khwarizmi, que floreció alrededor del 820". Asimismo, destaca que: "Los símbolos actuales > y < para "mayor que" y "menor que" se deben a Thomas Harriot. Los paréntesis redondos ( ) aparecen en 1544, y los paréntesis cuadrados [ ] y los corchetes \{ \} eran utilizados por Vietà hacía 1593" (STEWART, 2008, p. 73).

Ahora bien, dado a los escasos antecedentes histórico-epistemológicos específicos de la inecuación, en este estudio se desarrolló la idea de complejidad matemática a partir de la revisión de diversos textos matemáticos que presentan el tema de la inecuación (APOSTOL, 1961; LEITHOLD, 1998; LARSON et al., 1999; MARTINEZ et al., 2014). En base a dicha revisión, se sugiere un esquema (Figura 1) con todos los componentes que están involucrados en el estudio de dicho objeto matemático.

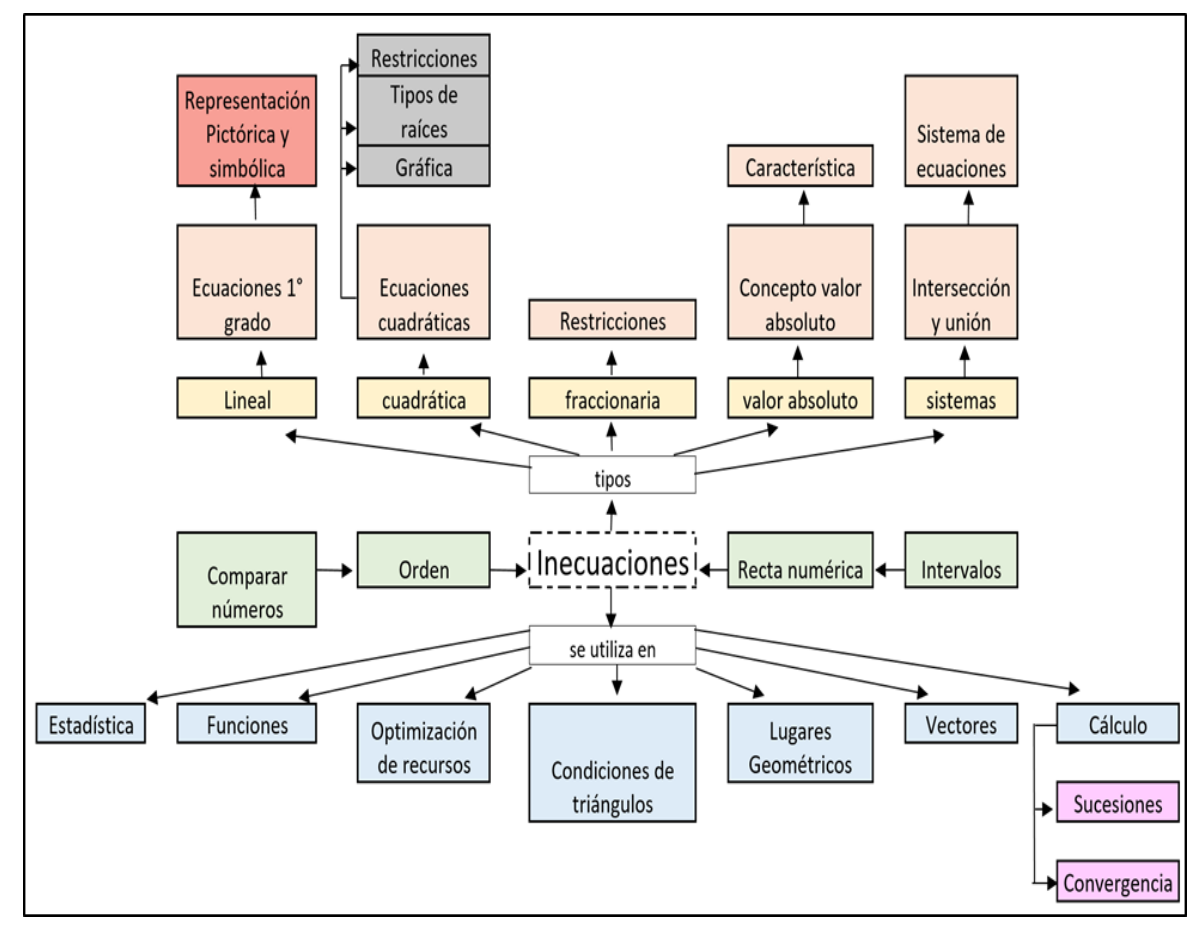

Figura 1 - Complejidad matemática de la inecuación Fuente: elaboración propia en base a la bibliografía consultada 
En el centro de la Figura 1 (de color verde) se presentan los conceptos previos para estudio de la inecuación (comparar números, orden, recta numérica e intervalos), los que darán a conocer la desigualdad de números, en el marco de diversos conjuntos numéricos y acorde a lo que se estipula en el currículum chileno para cada nivel; en cuarto, quinto y sexto año básico (10 a 12 años) se utiliza el conjunto de los números naturales, en séptimo año básico (13 años) los números enteros, en octavo año básico y primer año medio (14 y 15 años) números racionales, segundo año medio (16 años) números reales y en tercer y cuarto año medio (17 y 18 años) números complejos.

En el nivel superior de la Figura 1 (de color amarillo) se puede observar los tipos de inecuaciones (lineal, cuadrática, fraccionaria, valor absoluto y sistemas) con sus respectivos conceptos previos (de color naranjo), además de las especificaciones en las ecuaciones de primer grado y ecuaciones cuadráticas (de color rojo y gris). Finalmente, en el nivel inferior (de color celeste) se muestra dónde se utiliza la inecuación (estadística, funciones optimización de recursos, condiciones de triángulos lugares geométricos, vectores y cálculo).

Es importante destacar que las desigualdades son conceptos previos de las inecuaciones por lo que, en primera instancia, se define la desigualdad y sus axiomas de orden para, posteriormente, trabajarlas en la resolución de inecuaciones. Por ello, en los conceptos previos de la Figura 1 aparecen comparar números, orden, recta numérica e intervalos.

Esta propuesta permitiría determinar qué componentes de la inecuación están presentes en el currículum o textos escolares y, de esta manera, analizar la continuidad (o progresión) de dicho objeto matemático en el sistema escolar chileno lo que, según el Consejo Nacional de Profesores de Matemáticas (NCTM, 2000), es un análisis relevante dado que el tratamiento progresivo de un determinado objeto matemático es clave a la hora de valorar la excelencia de un currículum.

En esta línea, Pino-Fan et al. (2013), manifiestan la relevancia de verificar si los significados institucionales pretendidos son representativos del significado global de referencia de un determinado objeto matemático, puesto que la ausencia de alguno de sus componentes puede llevar a un tratamiento parcializado que puede afectar los procesos de enseñanza y aprendizaje.

\section{Metodología}

Para alcanzar el objetivo propuesto, analizar el currículum y los textos escolares chilenos para conocer las características del tratamiento de la inecuación durante la formación 
escolar y su relación con la complejidad matemática de dicho objeto en estudio, se empleó la técnica cualitativa de análisis de contenido.

En la primera etapa se analizó los siguientes programas de estudio: cuarto año básico (MINEDUC, 2013a), quinto año básico (MINEDUC, 2013b), sexto año básico (MINEDUC, 2013c), séptimo año básico (MINEDUC, 2016a), octavo año básico (MINEDUC, 2016b), primer año medio (MINEDUC, 2016c), segundo año medio (MINEDUC, 2011), tercer año medio (MINEDUC, 2015a) y cuarto año medio (MINEDUC, 2015b).

De dichos programas, tanto los objetivos de aprendizaje (OA) como los indicadores de evaluación, se convirtieron en unidades de análisis que fueron clasificadas basándonos en los componentes presentes en la Figura 1, con la finalidad de determinar cuáles de dichos componentes están presentes en el currículum escolar chileno. En una segunda etapa se procedió a analizar una colección de textos de estudio (de primaria y secundaria) que fueron distribuidos, gratuitamente, por el Ministerio de Educación de Chile, en el año 2016, en los establecimientos escolares que reciben subvención del estado.

Estos textos son: cuarto, quinto y sexto año básico (ESPINOZA; CANO, 2016a, 2016b, 2016c); séptimo año básico (MERINO et al., 2016); octavo año básico (CATALÁN et al., 2016); primer año medio (DEL VALLE; MUÑOZ; SANTIS, 2016); segundo año medio (MUÑOZ; RUPIN; JIMÉNEZ, 2016); tercer año medio (SAIZ; BLUMENTHAL, 2016) y cuarto año medio (MUÑOZ; GUTIÉRREZ; MUÑOZ, 2016).

Se trató de un análisis complementario al currículum, dado que el texto escolar es uno de los recursos más utilizado por los profesores a la hora de planificar sus clases. Por esta razón, se decidió analizar los textos correspondientes a los mismos niveles contemplados en el análisis curricular (de cuarto año básico a cuarto año medio), analizando un total de nueve textos. En esta etapa, la técnica de análisis utilizada es la propuesta por Cobo (citado en VÁSQUEZ; ALSINA, 2015) quien propone un análisis cualitativo que considera los siguientes pasos:

- Seleccionar los capítulos donde se aborda el tema de interés.

- Lectura minuciosa de los capítulos que tratan el tema. En nuestro caso, se clasificaron las unidades de análisis (actividades, ejercicios y/o problemas matemáticos) de acuerdo a su relación con los componentes de la Figura 1, la que adquiere el rol de instrumento para el análisis de datos.

- Elaboración de tablas comparativas que recogen los elementos de significados presentes en los textos analizados. 


\section{Análisis y discusión de los resultados}

A continuación se presentan los resultados obtenidos en el estudio. En primera instancia, se presentan los hallazgos del análisis curricular y, en segunda instancia, como un análisis complementario, se muestran los resultados obtenidos de los textos escolares analizados.

\subsection{Análisis del currículum chileno}

Para llevar a cabo el análisis del tratamiento de la inecuación en el currículum chileno seguimos dos pasos. El primer paso consistió en hacer una búsqueda en los programas de estudio de los distintos niveles para determinar cuáles son los niveles en los que se espera desarrollar el aprendizaje de la inecuación y qué se espera que los estudiantes aprendan en cada nivel y, en el segundo paso, relacionamos el tratamiento que se le da a la inecuación en el currículum chileno con la complejidad de dicho objeto matemático (propuesto en la Figura 1).

Cabe destacar que los planes y programas analizados son los entregados oficialmente por el Ministerio de Educación de Chile. Los documentos oficiales de cuarto, quinto y sexto año básico (rango etario de 10-12 años) fueron actualizados en el año 2012, los de séptimo y octavo año básico (rango etario de 13-14 años), en el año 2016, los de primero y segundo año medio (rango etario de 15-16 años), en el año 2011, y los de tercero y cuarto año medio (rango etario de 17-18años) se actualizaron en el año 2009, pero fueron implementados en el año 2014.

A continuación, en la Figura 2, se muestra la progresión del objeto matemático en estudio en el currículum escolar de Chile. En esta figura hemos destacado los OA que se relacionan directamente con las desigualdades e inecuaciones, los que se presentan como una línea de tiempo que permite ver la trayectoria del objeto matemático en estudio a través de los distintos cursos o niveles. 


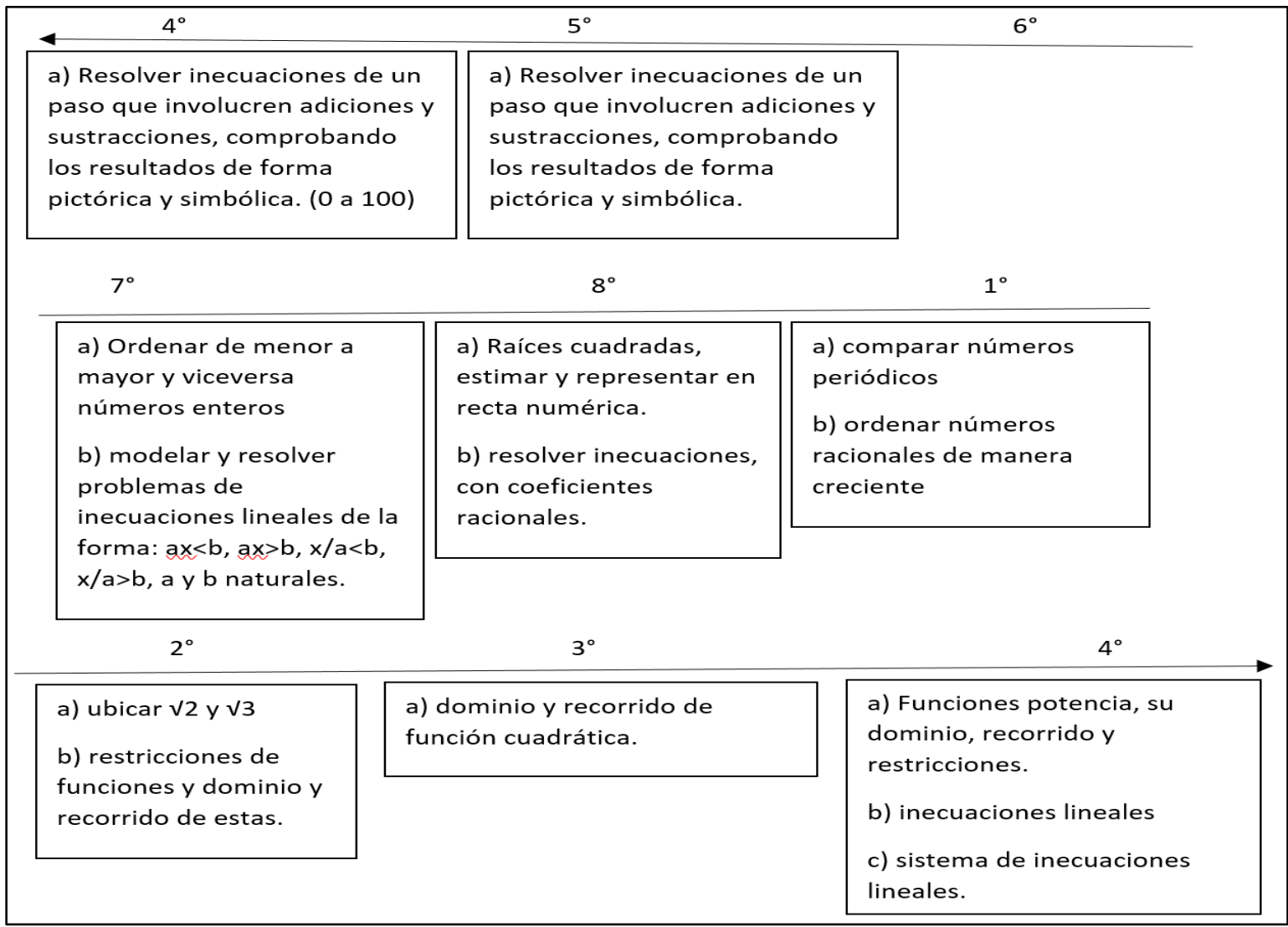

Figura 2 - Progresión del tratamiento de la inecuación en el currículum escolar chileno Fuente: elaboración propia

En la Figura 2 podemos ver que el objeto matemático en estudio (inecuaciones) se incorpora en el currículum desde cuarto año básico, trabajando en ese nivel y en quinto año básico de forma pictórica y simbólica directa. En el caso de sexto año básico no existe ningún objetivo o contenido que este directamente relacionado con la inecuación o desigualdad, por lo que no existe cuadro en la línea temporal.

Posteriormente, se incorpora el trabajo de la inecuación, de manera algebraica, en séptimo y octavo básico, aumentando el grado de dificultad en cuanto al conjunto numérico trabajado en cada curso (desde números enteros hasta números racionales). Por otra parte, vemos que desde primer año medio hasta tercer año medio la inecuación se encuentra presente como conocimiento previo para alcanzar otros OA, por lo tanto se observa una descontinuación del trabajo de inecuaciones como OA explícito. Finalmente, en cuarto año medio, podemos ver que se vuelve a retomar la inecuación como OA explícito, donde se propone un plan de formación similar al de séptimo y octavo año básico, agregando solo el contenido de sistema de inecuaciones.

Con la información entregada en la Figura 2, podemos ver que la actualización curricular a considerado una mayor continuidad en el estudio de la inecuación, sin embargo, como hemos señalado en el párrafo anterior, se observa una discontinuidad en el estudio explícito de dicho 
objeto matemático, observando un quiebre en la progresión o trayectoria de enseñanza, lo que podría afectar el aprendizaje de la inecuación. Dicho quiebre se evidencia cuando vemos que, en cuarto año medio, la unidad de inecuaciones es muy similar a la planteada en séptimo y octavo año básico, es decir, se vuelve a retomar su estudio explícito después de tres años de escolaridad incorporando los sistemas de inecuaciones lineales.

A continuación, en la Figura 3, se observa gráficamente la relación del currículum chileno con la propuesta de complejidad de la inecuación que se ha presentado en el marco referencial de este trabajo.

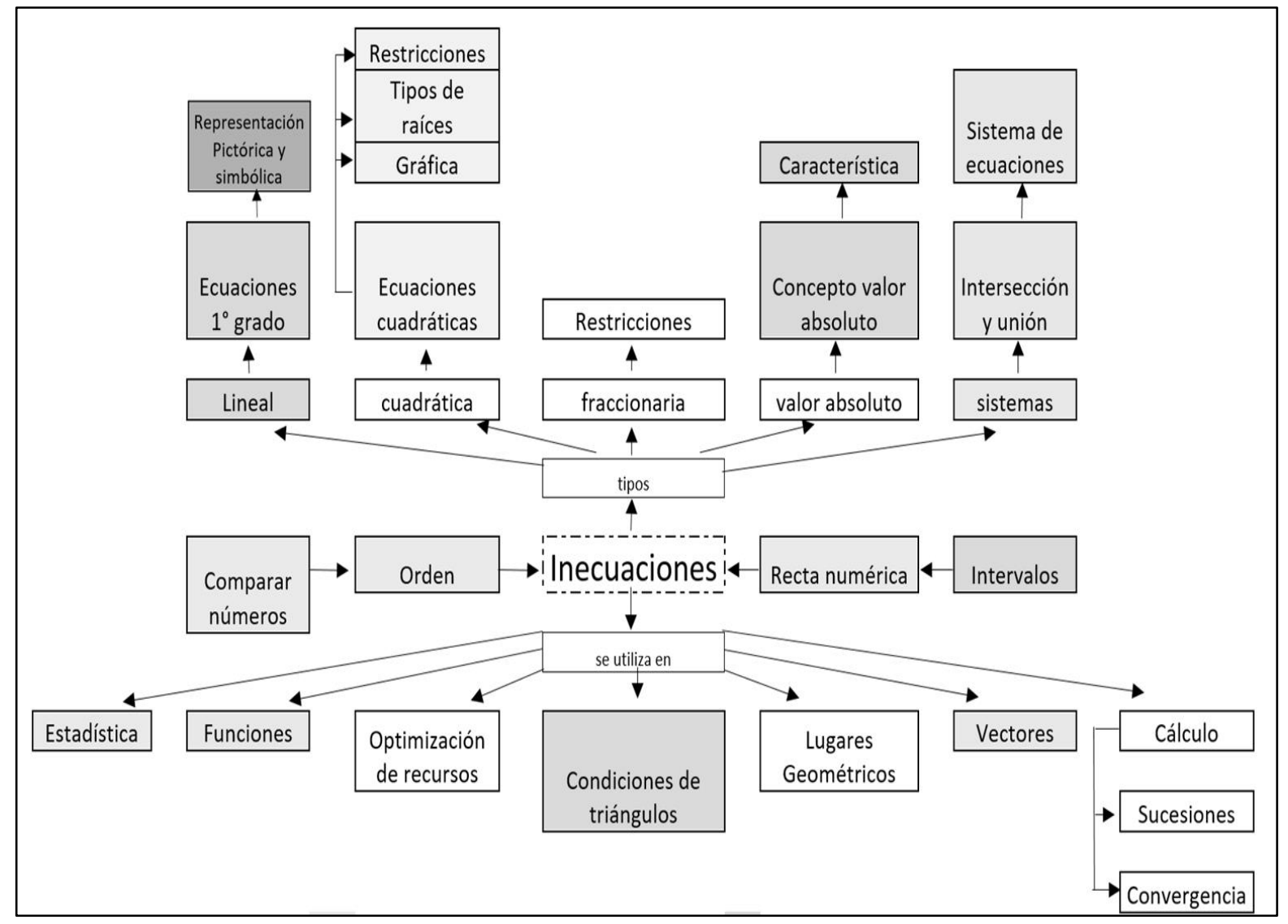

Figura 3 - Relación de currículum chileno con la complejidad de la inecuación Fuente: elaboración propia

En la Figura 3 se observa, de color gris, los contenidos que son abordados en el currículum chileno. De esta manera, se evidencia que dicho currículum no contempla el tratamiento de todos los componentes necesarios para el aprendizaje de la inecuación, pues no se considera tres tipos de inecuaciones:

- Inecuaciones cuadráticas: no están dentro del currículum, pero todo lo que es concepto previo (ecuaciones cuadráticas, restricciones y gráfica) está presente en tercer año medio, por lo que se considera posible incorporar su estudio en el programa de dicho nivel. 
- Inecuaciones fraccionarias: es un componente que requiere de un tratamiento muy similar al de inecuaciones cuadráticas. El aprendizaje previo de dicha inecuación sería la definición formal de una fracción y las restricciones de éstas, lo que se considera en el programa de estudio de segundo año medio. Por esta razón, se considera que este tipo de inecuación podría incorporarse en el currículum de ese nivel.

- Inecuaciones de valor absoluto: no se contempla dentro del currículum chileno, pero sus aprendizajes previos se ven en séptimo y octavo año básico, por lo tanto se considera que es posible incorporar el estudio de esta inecuación en primer año medio.

Dicho esto, si se considera que el énfasis a determinados componentes de la inecuación y el desconocimiento de otros (inecuaciones cuadráticas, fraccionarias y de valor absoluto) puede resultar en un cubrimiento epistémico parcializado, que puede afectar la idoneidad del proceso de enseñanza (PINO-FAN et al., 2013), resulta trascendental atender las ausencias observadas en los tres niveles mencionados (primero, segundo y tercer año medio).

\subsection{Análisis de los textos escolares 2016}

Tal como se ha señalado en el apartado de metodología, para analizar los textos se siguieron tres pasos. En el primero, se hizo una revisión de los textos de educación primaria y secundaria para seleccionar los capítulos donde se aborda el tema de interés ya sea de manera explícita (el título de capitulo anuncia su tratamiento), o bien, de manera implícita (en el capítulo hay algunas actividades donde se requiere de conocimiento previo sobre inecuación), encontrando un total de 35 capítulos de cuarto a sexto año básico, y 26 unidades desde séptimo año básico a cuarto año medio.

Esto nos permitió identificar cuáles son los niveles en los que se espera desarrollar el aprendizaje de la inecuación y qué se espera que los estudiantes aprendan en cada uno de los niveles. De esta manera, se pudo determinar que el objeto matemático en estudio estaba presente, de manera explícita, en los siguientes apartados de los textos de estudio: capítulo 6 del texto de cuarto año básico (10 años), capítulo 4 del texto de quinto año básico (11 años), unidad 2 sección 4 del texto de séptimo año básico (13 años), unidad 2 sección 5 del texto de octavo año básico (14 años) y en la unidad 2 del texto de cuarto año medio (18 años).

Posteriormente, el paso dos y tres (lectura minuciosa y elaboración de tablas comparativas) nos permitió conocer las actividades que se presentan en el texto y, a partir de ello, relacionar el tratamiento que se le da a la inecuación con la complejidad de dicho objeto matemático (propuesto en la Figura 1). 
Los textos de cuarto y quinto año básico son de la editorial Galileo Libros Ltda., del año 2016; en séptimo y octavo año básico son de la editorial SM Chile S.A., del año 2016, los de primero y segundo año medio corresponden a la misma editorial del periodo 2014-2016; el de tercer año medio corresponde a la editorial Cal y Canto, del periodo 2012-2016, y el de cuarto año medio corresponde a la editorial Santillana del Pacífico S. A., del periodo 2013-2016.

En la Figura 4, se destacan los OA detectados en el análisis de los capítulos y unidades seleccionadas, los que se presentan a través de una línea temporal en base a los cursos o niveles en que dichos $\mathrm{OA}$ son tratados. En dicha figura, se puede ver que el objeto matemático en estudio comienza a implementarse en el aula desde cuarto año básico (10 años) y, al igual que en quinto año básico (11 años), se trabaja el concepto de inecuación de manera pictórica y simbólica.

Posteriormente, en séptimo y octavo año básico (13 y 14 años), se comienza a trabajar la inecuación de manera algebraica y, en cuarto año medio (18 años), se retoma el estudio explícito de dicho objeto matemático. Esto nos permite dar cuenta que lo propuesto en los textos de estudio tiene estrecha relación con lo señalado en el currículum nacional, sin embargo, al analizar los textos podemos conocer el tipo de actividades presentes en los cursos (sexto año básico, primero, segundo y tercer año medio) en que la inecuación es considerada un conocimiento previo (ver detalles en el Cuadro 1).

Asimismo, la Figura 4 da cuenta que los textos escolares chilenos han incorporado el estudio de la inecuación de manera progresiva desde cuarto a octavo año básico (10 a 14 años), sin embargo, al igual que en el currículum, se descontinúa su estudio explícito hasta cuarto año medio (18 años), lo que afecta el carácter progresivo en el proceso de enseñanza y aprendizaje del objeto matemático en estudio. Por otra parte, al analizar el texto de cuarto año medio (18 años), específicamente, en la subunidad de sistemas de inecuaciones, nos encontramos con propuestas de ejercicios matemáticos en los que se trabaja el contenido de inecuaciones fraccionarias de un paso, lo que no está declarado en el currículum de dicho nivel. 


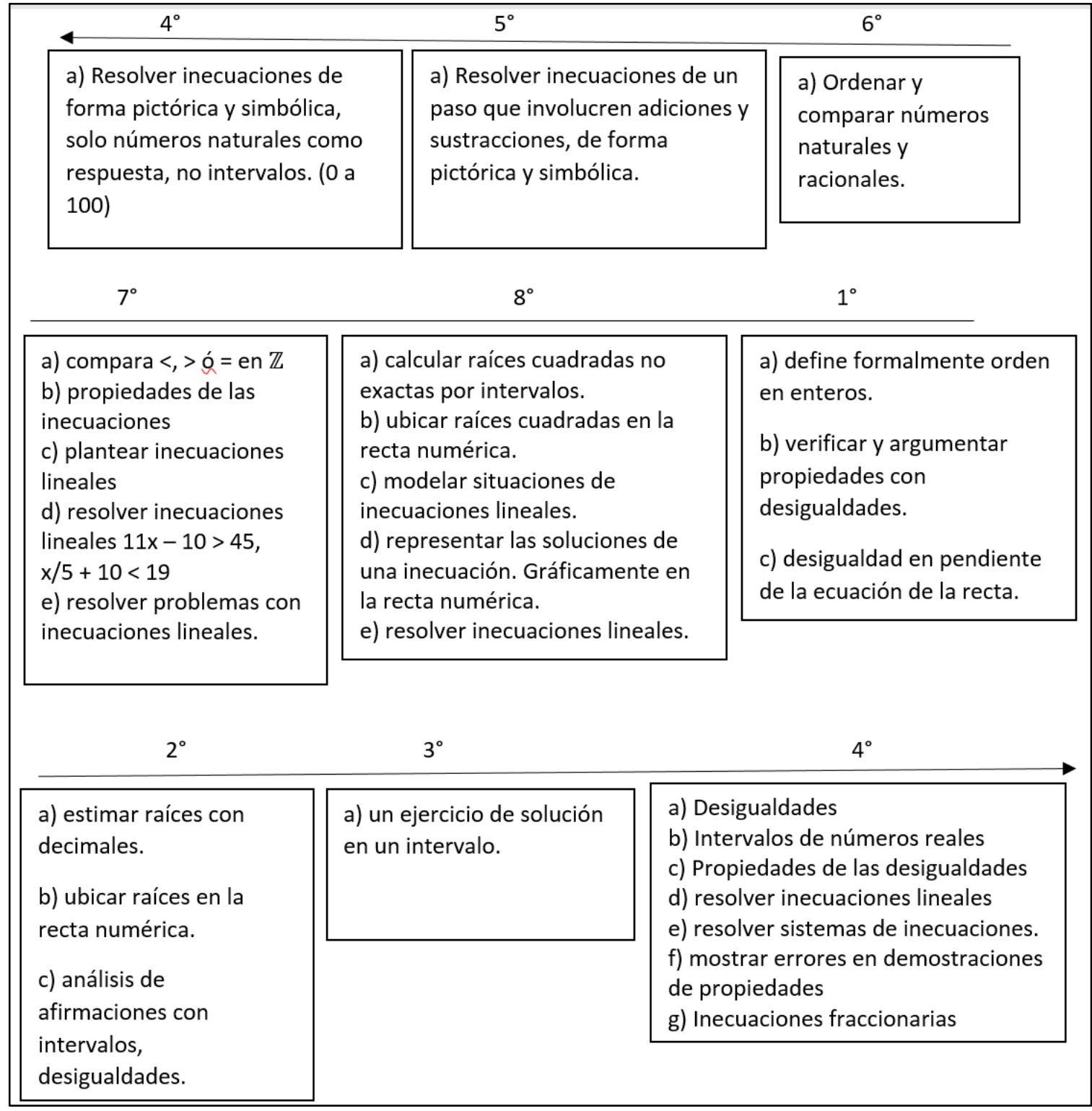

Figura 4 - Progresión del tratamiento de la inecuación en los textos escolares chilenos. Fuente: elaboración propia

A continuación, en el Cuadro 2, se presenta una selección de actividades propuestas en los textos escolares entregados por el Ministerio de Educación de Chile, en el año 2016. Para la construcción del dicho cuadro se recogieron actividades de cuarto año básico a cuarto año medio, lo que permite ver, tal como adelantamos en párrafos anteriores, el trabajo explicito (en cuarto, quinto, séptimo y octavo año básico, como también el caso de cuarto año medio) e implícito (en sexto año básico, primero, segundo y tercer año medio) del objeto matemático en estudio. 


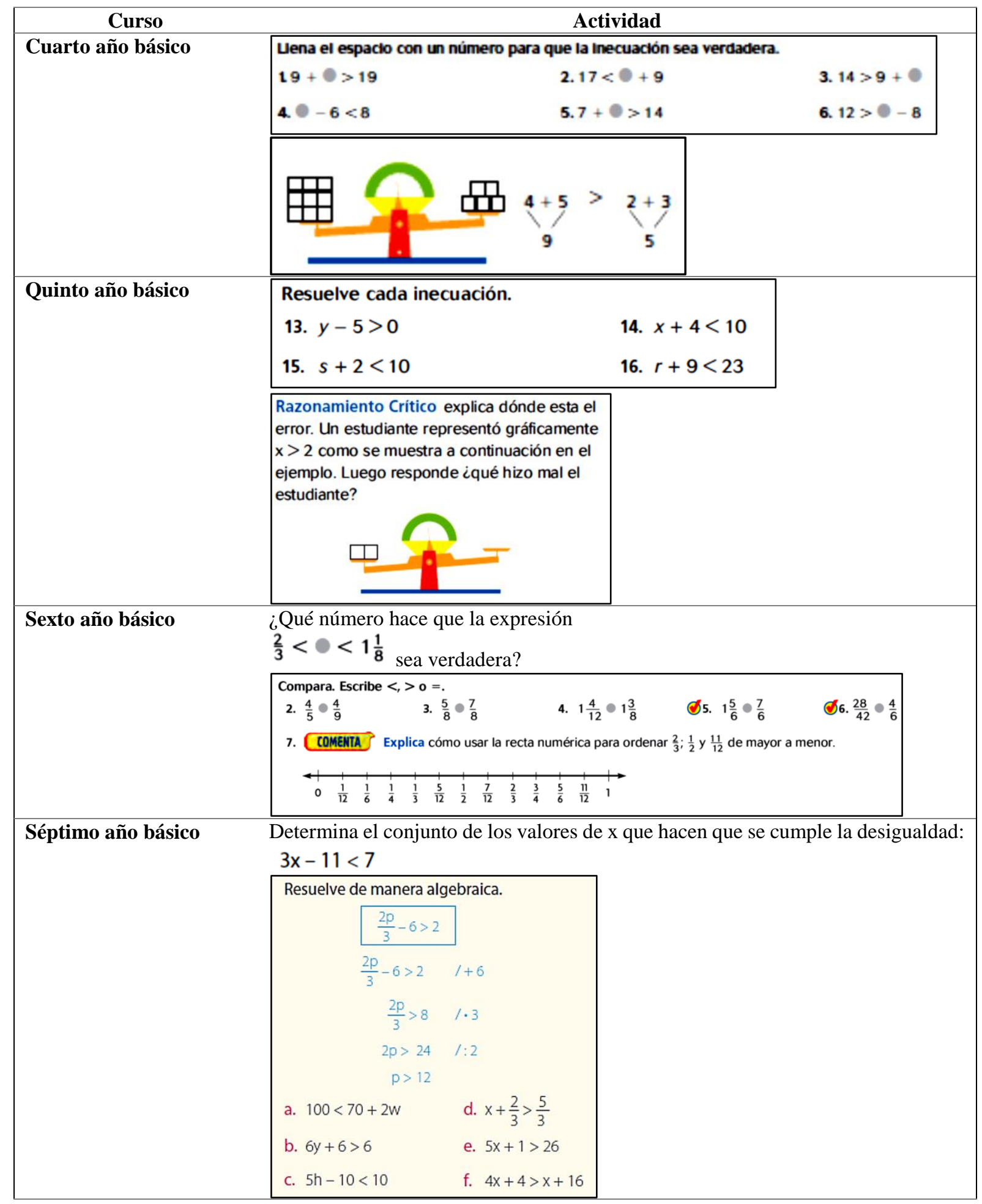




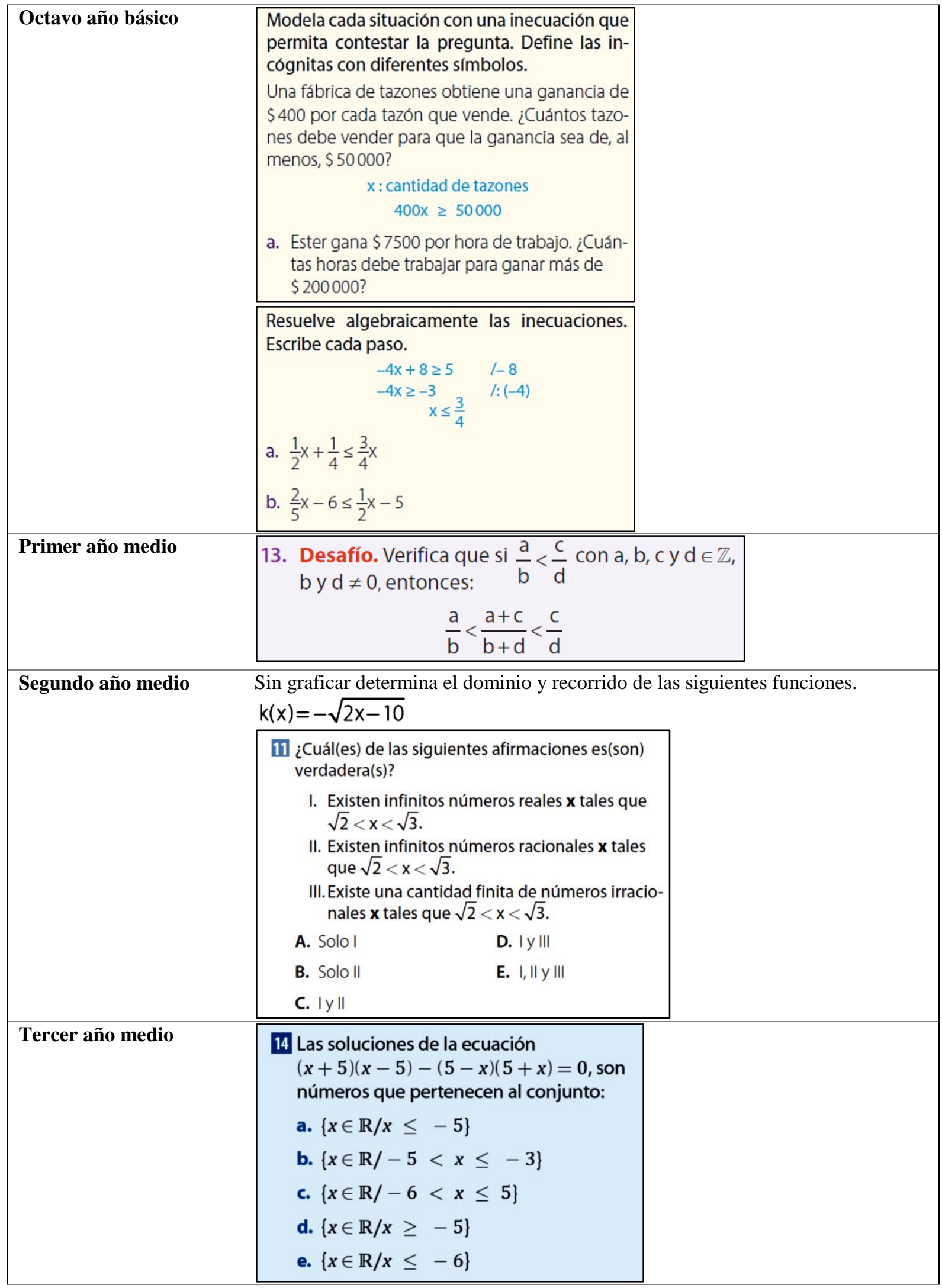




\begin{tabular}{|l|l|}
\hline Cuarto año medio & Resuelve los siguientes sistemas de inecuaciones con una incógnita. \\
& $\begin{array}{l}7 x+8>2-x \\
3 x+x^{2} \leqslant x^{2}-2 x \\
3 x-3 \geqslant 6 x+13\end{array}$ \\
& $\begin{array}{l}\text { Plantea los siguientes ejercicios como un sistema de inecuaciones lineales y } \\
\text { determina su conjunto solución. } \\
\frac{2 x+1}{3-x} \geqslant 0\end{array}$ \\
& S. SI $a>0$ y $b>0$, demuestra que $a+b>\frac{a^{2}+b^{2}}{a+b}$. \\
\hline
\end{tabular}

Cuadro 2 - Selección de actividades propuestas en textos escolares chilenos

Fuente: textos escolares otorgados por el Ministerio de Educación de Chile en el año 2016

Como se puede ver en el Cuadro 2, en cuarto año básico (10 años) la actividad pretende que los estudiantes comprendan el concepto de inecuación como una desigualdad donde se puede encontrar más de un resultado, para luego formalizarlo en quinto año básico (11 años). En séptimo y octavo año básico (13 y 14 años), van agregando dificultad a estas inecuaciones lineales, sin embargo, en primero, segundo y tercer año medio (16 y 17 años) la inecuación está presente en actividades muy puntuales y es considerada como un conocimiento previo. Finalmente, en cuarto año medio se retoma la unidad de inecuaciones (explícitamente), recordando todo lo trabajado hasta octavo año básico y agregando sistemas de inecuaciones con una incógnita, agregando ejercicios de inecuaciones fraccionarias.

A continuación, en la Figura 5, se observa gráficamente la relación de los textos escolares chilenos con la propuesta de complejidad de la inecuación.

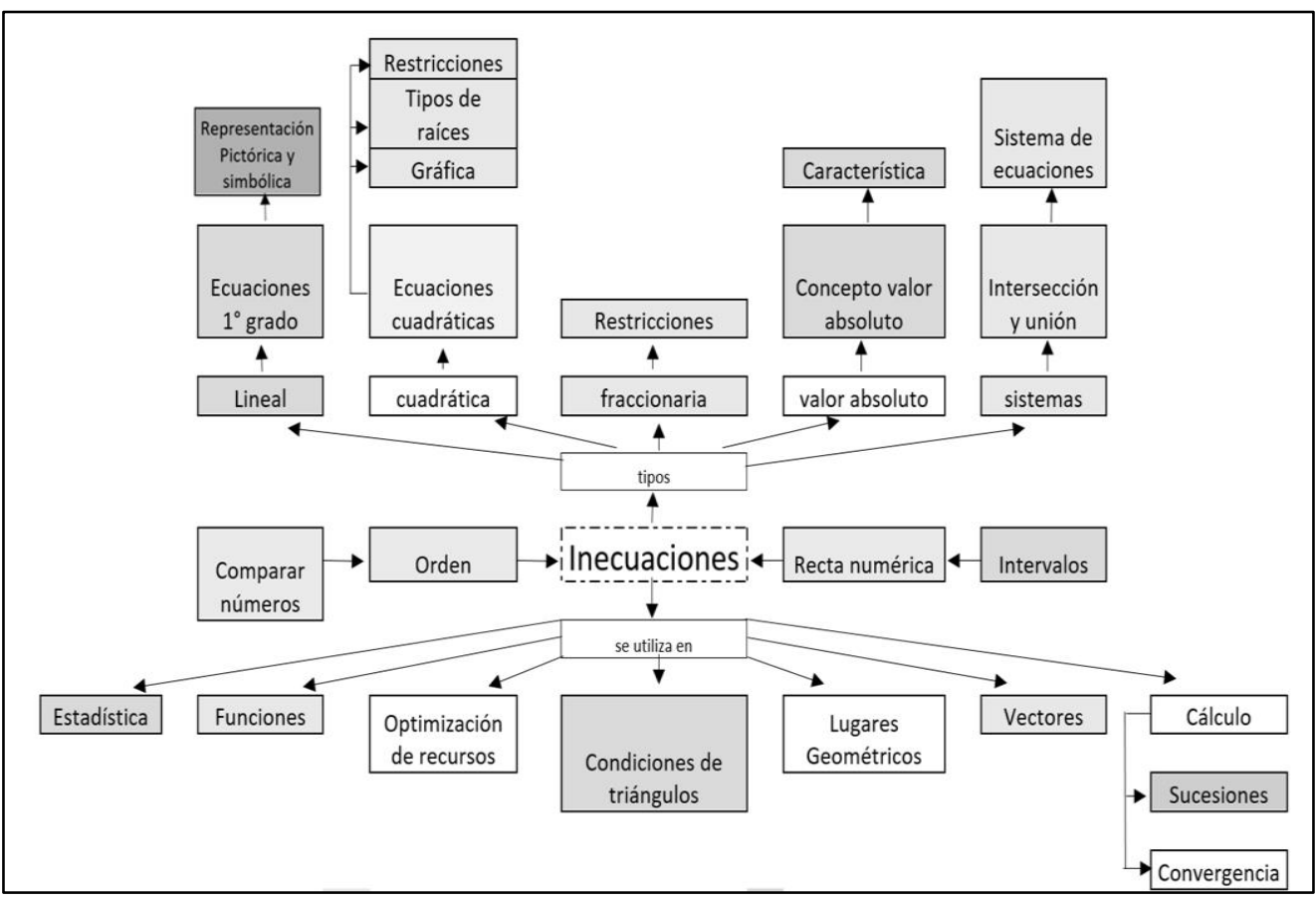

Figura 5 - Relación de los textos escolares chilenos con la complejidad de la inecuación Fuente: elaboración propia 
En la Figura 5 se asignó un color gris para destacar los componentes presentes en los textos escolares, de esta manera se puede observar que, de acuerdo a la Figura 1, el tratamiento de la inecuación no contempla todos los componentes, quedando ausente las inecuaciones cuadráticas, la inecuación valor absoluto y el desarrollo acabado de las inecuaciones fraccionarias. A la vez, podemos dar cuenta que el texto de estudio de cuarto año medio (18 años) contempla más contenidos en comparación a lo declarado en el currículum de dicho nivel, como es el caso de las inecuaciones fraccionarias.

\section{Consideraciones finales}

En base a la construcción de la Figura 1 (complejidad matemática de la inecuación) y a partir del análisis comparativo con el currículum nacional y los textos escolares otorgados por el Ministerio de Educación de Chile, se puede concluir que el tratamiento que se le otorga al objeto matemático en estudio (inecuaciones) no considera todos los componentes necesarios para la enseñanza de la inecuación a partir de su complejidad, pudiendo observar que tanto el currículum como los textos escolares dejan fuera las inecuaciones cuadráticas y las inecuaciones con valor absoluto, reconociendo, además, que se trabaja superficialmente las inecuaciones fraccionarias, lo que se observa en el análisis de los textos escolares.

A la vez, damos cuenta que en los niveles estudiados (cuarto año básico a cuarto año medio) no existe una continuidad o progresión en el tratamiento de la inecuación, pues en el currículum se observa que hay niveles donde la inecuación no es considerada de manera explícita (sexto año básico, primero, segundo y tercer año medio), reconociendo los mismos hallazgos en el análisis de los textos escolares, donde pudimos observar que en los cursos donde se trata la inecuación de manera implícita, se presentan actividades que requieren del manejo del objeto matemático en estudio para lograr comprender un objeto matemático nuevo, por ejemplo, para trabajar el concepto de dominio y recorrido de diferentes funciones.

Cabe destacar que los programas analizados, que componen el currículum chileno, se implementaron en el año 2016, y que en séptimo y octavo año básico hubo un cambio significativo al incorporar la enseñanza de la inecuación. Es por ello que la discontinuidad que se produce en los niveles de primero, segundo y tercero año medio es preocupante dado que, actualmente, los estudiantes que cursan primer año medio fueron formados con un programa que no contemplaba la enseñanza de dicho objeto matemático, por lo tanto, no están preparados para trabajar en unidades de estudio donde la inecuación es parte del conocimiento previo para 
iniciar el estudio de otras materias. Conociendo este escenario, resulta problemático que en los ajustes curriculares, propuestos para el año 2017 en los cursos de primero y segundo año medio, no se incorporé la enseñanza explícita de inecuaciones.

Por otra parte, la escasa información que se logra obtener para reconstruir el significado global de la inecuación (BORELLO; LEZAMA, 2009) implica una limitante en este estudio, pues la propuesta de la Figura 1 se presenta como una reconstrucción en desarrollo que puede ser ampliada a partir de los hallazgos de nuevos estudios en esta temática.

Asimismo, es importante destacar que, en relación a los textos escolares, este estudio se limitó al análisis de actividades y definiciones para hacer las relaciones permitentes con los componentes de la Figura 1. Sin embargo, próximos estudios podrían profundizar el análisis epistémico desde un punto de vista pragmatista (FONT; GODINO; GALLARDO, 2013), considerando otros aspectos como: propiedades y teoremas, argumentos y otras definiciones presentes.

Dado el panorama actual del tratamiento de las inecuaciones en el currículum y textos escolares chilenos, surgen las siguientes cuestiones cómo posibles problemáticas a investigar: ¿Cómo afecta la discontinuidad en la enseñanza de la inecuación a los estudiantes chilenos? ¿Cómo se desarrolla este objeto matemático en el currículum de otros países?

\section{Agradecimientos}

Estudio realizado en el marco del proyecto PMI UCM1310.

\section{Referencias}

APOSTOL, T. Calculus. 2. ed. Barcelona-Bogotá-Buenos Aires-Caracas-México: Editorial Reverte S.A, (No. 517 A6), 1961.

BALDOR, A. Álgebra. 5. ed. Mexico: Ed. Editorial Patria, 1988.

BORELLO, M; LEZAMA, J. Hacia una resignación de las desigualdades e inecuaciones a partir de las prácticas del profesor. En: LESTÓN, P (Ed.). Acta Latinoamericana de Matemática Educativa. Comité Latinoamericano de Matemática Educativa. México, Coacalco, v. 22, 2009. p. 1091-1099.

BREDA, A.; FONT, V.; PINO-FAN, L. R. Criterios valorativos y normativos en la Didáctica de las Matemáticas: el caso del constructo idoneidad didáctica. Bolema, Rio Claro, en prensa.

BREDA, A.; LIMA, M. R. Estudio de caso sobre el análisis didáctico realizado en un trabajo final de un máster para profesores de matemáticas en servicio. REDIMAT - Journal of Research in

Mathematics Education, España, v. 5, n. 1, p. 74-103, 2016. 
BREDA, A.; FONT, V.; LIMA, V. M. R. A noção de idoneidade didática e seu uso na formação de professores de matemática. Jornal Internacional de Estudos em Educação Matemática, Brasil, v. 8, p. 4-41, 2015.

BREDA, A.; PINO-FAN, L.; FONT, V. Meta didactic-mathematical knowledge of teachers: criteria for the reflection and assessment on teaching practice. EURASIA Journal of Mathematics, Science and Technology Education, Turquía, v. 13, n. 06, p. 1893-1918, 2017.

CATALÁN, D. et al. Textos del estudiante Matemática $8^{\circ}$ básico. Santiago, Chile: Editorial SM Chile S.A, 2016.

CERDA, G. et al. Fortalecimiento de competencias matemáticas tempranas en preescolares, un estudio chileno. Psychology, Society \& Education, España, Almería., v.3, n.1, p. 23-39, 2011.

DEL VALLE, J.; MUÑOZ, G.; SANTIS, M. Textos del estudiante Matemática $1^{\circ}$ medio. 3 . ed. Santiago, Chile: Editorial SM Chile S.A, 2016.

ESPINOZA, Y.; CANO, S. Texto del estudiante Matemática $4^{\circ}$ básico. 3. ed. Santiago, Chile: Editorial Galileo Libros Ltda., 2016a.

ESPINOZA, Y.; CANO, S. Texto del estudiante Matemática $5^{\circ}$ básico. 3. ed. Santiago, Chile: Editorial Galileo Libros Ltda, 2016b.

ESPINOZA, Y.; CANO, S. Texto del estudiante Matemática $6^{\circ}$ básico 3. ed. Santiago, Chile: Editorial Galileo Libros Ltda, 2016c.

FONT, V.; BREDA, A.; SECKEL, M. J. Algunas implicaciones didácticas derivadas de la complejidad de los objetos matemáticos cuando estos se aplican a distintos contextos. Revista Brasileira de Encino de Ciencias e tecnología RBECT, Brasil, v. 10, n. 2, p. 1-23, 2017.

FONT, V.; GODINO, J. D.; GALLARDO, J. The emergence of objects from mathematical practices. Educational Studies in Mathematics, Países bajos, Dordrecht, v. 82, p. 97-124, 2013.

FONT, V.; PLANAS, N.; GODINO, J. D. Modelo para el análisis didáctico en educación matemática. Infancia y Aprendizaje, España, v. 33, n. 1, p. 89-105, 2010.

GARROTE, M.; HIDALGO, M.; BLANCO, L. Dificultades en el aprendizaje de las desigualdades e inecuaciones. Revista Suma, España, Catalunya, v. 46, p. 37-44, 2004

GODINO, J. D. Teoría de las Funciones Semióticas. Un enfoque ontológico semiótico de la cognición e instrucción matemática. Trabajo de investigación presentado para optar a la Cátedra de Universidad de Didáctica de la Matemática de la Universidad de Granada. Noviembre, 2003.

GODINO, J. D. et al. Análisis y valoración de la idoneidad didáctica de procesos de estudio de las matemáticas. Paradigma, Maracay, v. 27, n. 2, p. 221-252, 2006.

GODINO, J. D. et al. Pauta de análisis y valoración de la idoneidad didáctica de procesos de enseñanza y aprendizaje de la matemática. Documento no publicado del Departamento de Didáctica de la Matemática. Universidad de Granada, 2007. Disponible en: <http://www.ugr.es/local/jgodino/indice_eos.htm>. Acceso en: 11 jun 2018.

GODINO, J. D. Indicadores de la idoneidad didáctica de procesos de enseñanza y aprendizaje de las matemáticas. Cuadernos de Investigación y Formación en Educación Matemática, Costa Rica, v. 8, n. 11, p. 111-132, 2013. 
GODINO, J. D.; WILHELMI M. R.; BENCOMO, D. Suitability criteria for a mathematical instruction process. A teaching experience with the function notion. Mediterranean Journal for Research in Mathematics Education, Nicosia, v. 4, n. 2, p. 1-26, 2005. Disponible en: <http://www.ugr.es/ j godino/articulos_ingles/suitability_criteria_functions.pdf>. Acceso en: 11 jun 2018.

LARSON, R. et al. Cálculo y geometría analítica. 6. ed. España: Editorial S.A. MCGRAW-HILL., 1999.

LEITHOLD, L. El cálculo. 7. ed. México: Editorial de Oxford University Press. Edición Número 517,1998 .

MARTINEZ, F. et al. Recursos para la Formación Inicial de Profesores de Enseñanza Básica, Álgebra. Proyecto FONDEF - CONICYT D09 I1023 (2011 - 2014), 2014. 130 p.

MERINO, R. et al. Textos del estudiante Matemática $7^{\circ}$ básico. 1. ed. Santiago, Chile: Editorial SM Chile S.A, 2016.

MINEDUC. Programa de estudio cuarto año básico. 3. ed. Santiago, Chile: Unidad de currículum y evaluación, 2013a. Disponible en: <http://www.curriculumenlineamineduc.cl/605/articles18979_programa.pdf>. Acceso en: 23 oct 2018.

MINEDUC. Programa de estudio quinto año básico. 3. ed. Santiago, Chile: Unidad de currículum y evaluación, 2013b. Disponible en: <http://www.curriculumenlineamineduc.cl/605/articles18980_programa.pdf>. Acceso en: 23 oct 2018.

MINEDUC. Programa de estudio sexto año básico. 3. ed. Santiago, Chile: Unidad de currículum y evaluación, 2013c. Disponible en: <http://www.curriculumenlineamineduc.cl/605/articles18981_programa.pdf>. Acceso en: 23 oct 2018.

MINEDUC. Programa de estudio séptimo básico. 1. ed. Santiago, Chile: Unidad de currículum y evaluación, 2016a. Disponible en: <http://www.curriculumenlineamineduc.cl/605/articles18982_programa.pdf>. Acceso en: 23 oct 2018.

MINEDUC. Programa de estudio octavo básico. 1. ed. Santiago, Chile: Unidad de currículum y evaluación, 2016b. Disponible en: 〈http://www.curriculumenlineamineduc.cl/605/articles18983_programa.pdf>. Acceso en: 23 oct 2018.

MINEDUC. Programa de estudio primer año medio. 1. ed. Santiago, Chile: Unidad de currículum y evaluación, 2016c Disponible en:<http://www.curriculumenlineamineduc.cl/605/articles34359_programa.pdf>. Acceso en: 23 oct 2018.

MINEDUC. Programa de estudio segundo año medio. 5. ed. Santiago, Chile: Unidad de currículum y evaluación, 2011. Disponible en: <http://www.curriculumenlineamineduc.cl/605/articles34360_programa.pdf>. Acceso en: 23 oct 2018.

MINEDUC. Programa de estudio tercer año medio. 2. ed. Santiago, Chile: Unidad de currículum y evaluación, 2015a. Disponible en: 〈http://www.curriculumenlineamineduc.cl/605/articles-34361_pr ograma.pdf $>$. Acceso en: 23 oct 2018.

MINEDUC. Programa de estudio cuarto año medio. 2. ed. Santiago, Chile: Unidad de currículum y evaluación, 2015b. Disponible en: <http://www.curriculumenlineamineduc.cl/605/articles-34362_ programa.pdf $>$. Acceso en: 23 oct 2018.

MUÑOZ, G.; GUTIÉRREZ, V.; MUÑOZ, S. Matemática IV medio Texto del estudiante. 3ra. ed. Santiago, Chile: Editorial Santillana del Pacífico S. A, 2016. 
MUÑOZ, G.; RUPIN, P.; JIMÉNEZ, L. Matemática $2^{\circ}$ medio. 2. ed. Santiago, Chile: Editorial SM Chile S.A, 2016.

NATIONAL COUNCIL OF TEACHERS OF MATHEMATICS. Principles and standards for school mathematics. 1. ed. Reston, VA: NCTM, 2000.

PARRA, Y.; PINO-FAN, L. R. Análisis ontosemiótico de libros de texto chilenos: el caso del concepto de función. En: CONTRERAS, J. M. et al. (Eds.). CONGRESO INTERNACIONAL VIRTUAL SOBRE EL ENFOQUE ONTOSEMIÓTICO DEL CONOCIMIENTO Y LA INSTRUCCIÓN MATEMÁTICOS, 2do congreso, 2017. España. Actas del Segundo Congreso Internacional Virtual sobre el Enfoque Ontosemiótico del Conocimiento y la Instrucción Matemáticos. Granada: Universidad de Granada, 2017. Disponible en: <http://enfoqueontosem iotico.ugr.es/civeos/parra.pdf>. Acceso en: 11 jun 2018.

PAULOS, J. El hombre anumérico: El analfabetismo matemático y sus consecuencias. 1. ed. Barcelona: Editorial Tusquets, 1990.

PINO, J.; BLANCO, L. Análisis de los problemas de los libros de texto de matemáticas para alumnos de 12 a 14 años de edad de España y de chile en relación con los contenidos de proporcionalidad. Publicaciones, España, Granada, v. 38, p. 63-88, 2008.

PINO, L. et al. Idoneidad epistémica del significado de la derivada en el currículo de bachillerato, Paradígma, Maracay, v. 34, n. 2, p. 123-150, dic. 2013.

RICO, L. Complejidad del currículo de matemáticas como herramienta profesional. Revista latinoamericana de investigación en matemática educativa, Colombia, v. 1, n. 1, p. 22-39, mar. 1998.

RONDERO, C.; FONT, V. Articulación de la complejidad matemática de la media aritmética. Enseñanza de las Ciencias, España, v. 33, n. 2, p. 29 - 49, jun. 2015.

SAIZ, O.; BLUMENTHAL, V. Texto del estudiante Matemática $3^{\circ}$ medio. 3. ed. Santiago, Chile: Editorial Cal y Canto, 2016.

SECKEL, M. J. Competencia en análisis didáctico en la formación inicial de profesores de educación básica con mención en matemática. 2016. 291f. Tesis (Doctorado en Formación del profesorado: práctica educativa y comunicación) - Universitat de Barcelona, Barcelona, 2016.

STEFFENS, K. The History of Approximation Theory: From Euler to Bernstein. 1ra. ed. Boston: Birkhauser, 2006.

STEWART, I. Historia de las matemáticas en los últimos 10.000 años. 1. ed. España, Barcelona: Editorial Crítica, S.L., 2008. (Publicación original en 2007).

VARGAS, A. Propuesta para la enseñanza y aprendizaje de las inecuaciones lineales. Revista educación, Costa Rica, v.37, n. 2, p. 1-16, jul. 2013.

VÁSQUEZ, C.; ALSINA, A. Un modelo para el análisis de objetos matemáticos en libros de textos chileno: situaciones problemáticas, lenguaje y conceptos sobre probabilidad. Profesorado: revista de currículum y formación de profesorado, España, v. 19, n. 2, p. 441- 462, may. 2015.

VRANCKEN, S.; ENGLER, A.; MÜLLER, D. Inecuaciones algebraicas. Una experiencia didáctica articulando diversos sistemas de representación. Yupana, Argentina, v. 10, n. 5, p. 55-66, mar. 2010. 
Submetido em 09 de Maio de 2017. Aprovado em 21 de Novembro de 2017. 